University of Nebraska - Lincoln

DigitalCommons@University of Nebraska - Lincoln

Publications from USDA-ARS / UNL Faculty

U.S. Department of Agriculture: Agricultural

Research Service, Lincoln, Nebraska

6-27-2017

\title{
Honey bee gut dysbiosis: a novel context of disease ecology
}

Kirk E. Anderson

Vincent A. Ricigliano

Follow this and additional works at: https://digitalcommons.unl.edu/usdaarsfacpub

This Article is brought to you for free and open access by the U.S. Department of Agriculture: Agricultural Research Service, Lincoln, Nebraska at DigitalCommons@University of Nebraska - Lincoln. It has been accepted for inclusion in Publications from USDA-ARS / UNL Faculty by an authorized administrator of DigitalCommons@University of Nebraska - Lincoln. 


\section{Honey bee gut dysbiosis: a novel context of disease ecology Kirk E Anderson ${ }^{1,2}$ and Vincent A Ricigliano ${ }^{1}$}

\begin{abstract}
The honey bee microbiota has become a hot-spot of recent research. Highly co-evolved with its host, the hindgut microbiota of a worker honey bee consists of six bacterial species shown to occur reliably in particular proportions. Altered microbiota structure is associated with host deficiencies, and a variety of bacteria found throughout the hive environment can dominate the worker gut suppressing or displacing microbiota function. The synthesis presented here suggests environmental insults alter gut bacterial balance, leading to decreased host function and disease progression. Specific functional groups of native bacteria represent a model system to investigate dysbiosis and the evolution of host tolerance/resistance traits in honey bee-microbe interactions.
\end{abstract}

\begin{abstract}
Addresses
${ }^{1}$ USDA-ARS Carl Hayden Bee Research Center, Tucson, AZ 85719, United States

${ }^{2}$ Department of Entomology and Center for Insect Science, University of Arizona, Tucson, AZ 85721, United States

Corresponding author: Anderson, Kirk E (kirk.anderson@ars.usda.gov)
\end{abstract}

Current Opinion in Insect Science 2017, 22:125-132

This review comes from a themed issue on Social insects

Edited by Amy Toth and Adam Dolezal

For a complete overview see the Issue and the Editorial

Available online 27th June 2017

http://dx.doi.org/10.1016/j.cois.2017.05.020

2214-5745/Published by Elsevier Inc.

\section{Introduction}

Biology is embracing a paradigm shift that incorporates beneficial bacterial function into general animal health. Animals are now considered a coordinated and cooperative set of genes, many of which are bacterial in origin. While microbes were historically cast as disease agents, 'omics' technology has facilitated the understanding of individuals as holobiont: the macro-organism plus its microbiota $\left[1^{\circ}\right]$. The microbiota is the entire community of microbes associated with a multicellular organism from pathogen to mutualist. The evolution and function of microbiota involves arguments that range from disease ecology to group selection to host niche fidelity $\left[2,3^{\bullet \bullet}\right]$. The animal gut is a particularly desirable microbial niche, providing a highly consistent source of nutrients and tissue types. This predictable niche facilitates microbial community structure via many evolutionary mechanisms including microbial cooperation and competition, host tolerance and host resistance to microbial colonization. The resultant gut microbiota can influence multiple host systems, and provide host protection via competitive exclusion of opportunists and pathogens. In this review we discuss the bacterial microbiota of a healthy honey bee gut and reconcile the occurrence and prevalence of hive and gut bacteria with reference to gut bacterial establishment and succession, bacterial community structure and dysbiosis. We synthesize evidence for the progression of gut dysbiosis and provide a novel context of honey bee disease ecology.

Chronic disease states involving host-microbial dysfunction have become a focal point of medical research [4]. Constituent or 'core' gut microbiota is hypothesized to mediate susceptibility to disease and ultimately even shape the ecology and evolution of disease states [5]. The honey bee provides a model system for understanding the relationship between microbiota and disease ecology because its' gut microbiota is highly predictable, cultivable, spatially structured, taxonomically simple, and reliably manipulated [6]. The honey bee gut microbiota affects host endocrine and immune signaling, disease susceptibility, growth and development, nutrition and behavior among others (Table 1). Recent investigations have revealed a honey bee gut microbiota with strict membership and structure, and simultaneously exposed a variety of alternate enterotypes or dysbiotic states (Figure 1). In stark contrast to humans and Drosophila, the honey bee hindgut contains a highly consistent bacterial community of six omnipresent species clusters and a few others more often found with deeper sequencing (Figure 1). These six species clusters comprise the core gut bacteria, a robust ecosystem integrated with host physiology $\left[7^{\bullet}, 8,9^{\bullet \bullet}, 10\right]$. While the evenness of the gut community has been proposed as a measure of healthy host physiology [11], healthy or 'core' community structure is an ongoing argument, because studies vary in their approaches [12], and community structure seems to alter predictably with adult age (Figure 1). Despite this variation, next generation sequencing (16S amplicons) of whole worker guts of Apis mellifera from around the world are remarkably consistent in structure and membership, providing strong insights into the core gut bacterial community $[8,13]$.

The honey bee hive contains a variety of 'fringe' niches that support bacterial species not typically found in the worker gut or pollination environment. These fringe 


\begin{tabular}{|c|c|c|c|c|}
\hline \multicolumn{5}{|c|}{ A select list of functions associated with honey bee associated gut microbiota. } \\
\hline Inferred or demonstrated function & Bacteria & Host niche ${ }^{a}$ & Citation & Approach \\
\hline Host adhesion/biofilm formation & Snodgrassella alvi & Gut/ileum & Powell et al. [19^0] & $\begin{array}{l}\text { Mutagenesis/genomic } \\
\text { screen }\end{array}$ \\
\hline Protection from opportunists & S. alvi & Gut/ileum & Maes et al. [22*0] & Host feeding treatment \\
\hline Immune cascade and melanization & Frischella perrara & Ileum/pylorus & Engel et al. $\left[27^{\bullet \bullet}\right]$ & Host feeding treatment \\
\hline Stimulation of adult immune response & Gut community, S. alvi & Hindgut & Kwong et al. $\left[17^{\bullet \bullet}, 2^{\circ}\right]$ & Host feeding treatment \\
\hline Differential adult immune response & F. perrara, S. alvi & lleum/pylorus & Emery et al. $\left[43^{\circ}\right]$ & Host feeding treatment \\
\hline Enriched carbohydrate metabolism & Gut community & Hindgut & $\begin{array}{l}\text { Engel et al. [29], } \\
\text { Lee et al. [30] }\end{array}$ & $\begin{array}{l}\text { Gut bacteria metabolic } \\
\text { profiling }\end{array}$ \\
\hline Digestion of complex carbohydrates & Gilliamella apicola & Gut/ileum & Engel et al. [29] & $\begin{array}{l}\text { Overlay agar containing } \\
\text { PGA }\end{array}$ \\
\hline Metabolism of toxic sugars & G. apicola & Gut/ileum & Zheng et al. [52] & carbon utilization assay \\
\hline Increased larval survival & $\begin{array}{l}\text { Parasaccharibacter } \\
\text { apium }\end{array}$ & $\begin{array}{l}\text { Hive/larvae/queen } \\
\text { gut }\end{array}$ & Corby-Harris et al. [16,41] & Larval supplementation \\
\hline Pioneer species early colonization & Lactobacillus firm5 & $\begin{array}{l}\text { Hive/larvae/all } \\
\text { castes }\end{array}$ & Anderson et al. [38] & Host feeding treatment \\
\hline Insulin signaling & Gut community & Hindgut & Zheng et al. [10] & Host feeding treatment \\
\hline Growth and development & Gut community & Hindgut & $\begin{array}{l}\left.\text { Maes et al. [22 }{ }^{\circ}\right] \text {, } \\
\text { Zheng et al. [10] }\end{array}$ & Host feeding treatment \\
\hline
\end{tabular}

environments are populated by core hive bacteria; adapted to survive the most extreme hive environments (Figure 1). In the context of health and disease, functional roles of these bacteria are not easily reduced to beneficial or detrimental $\left[1^{\circ}\right]$. Drawing parallels with the human microbiome, core hive bacteria can be considered analogous to the collective skin and oral microbiome of humans, comprised of protective opportunists, lurking pathogens, and some that function by context [14]. A subset of this group occurs consistently across various hive niches including food storage, mouth parts, nurse glands and queen guts (Figure 1). Dominant among core hive bacteria are Parasacharribacter apium and Lactobacillus kunkeei. First noted for their ability to grow in honey-rich conditions, both of these bacteria also exploit highly antimicrobial royal jelly, a testament to their co-evolved hive nature $[15,16]$. These two species are constitutive to the hive, but have close relatives that inhabit floral nectar and the guts of solitary and social bees $\left[17^{\bullet \bullet}, 18\right]$. L. kunkeei is fond of any niche high in fructose or oxygen, likely ever present on the biotic and abiotic fringes of the honey processing factory (Figure 1). P. apium is more abundant in behavioral networks associated with royal jelly, and is highly oxygen tolerant, possessing a suite of genes to process reactive oxygen species (ROS). An informal genome comparison suggests complementary strategies, but both species are able to deal with fluctuating oxygen levels predicted for the gut $\left[20^{\circ}\right]$, and both are detected in the worker gut when the host has been stressed [11]. The prevalence of these bacteria in the worker gut is associated with disease states in social bees and is negatively correlated with the size of the native bacterial community suggesting they diminish microbiota structure and function $\left[9^{\bullet \bullet}, 11,21,22^{\bullet \bullet}, 23,24^{\bullet}\right]$.

\section{Gut microbiota structure and function}

Guts contain biofilms, defined as a stratified matrix of bacteria that assembles via adhesion to the host epithelium and the production of extrapolymeric substances. This bacterial layer occurs at the molecular interface between host physiology and environment and plays an integral role in information exchange. Ideally, natural selection would choose a protector or multi-species biofilm according to the greatest collection of beneficial defensive traits [25]. More proximally, host gut function is reliant on biofilm structure. A biofilm occurs in the honey bee ileum, an expandable constriction between the midgut and rectum where Snodgrassella alvi interfaces with host tissue and is overlaid by Gilliamella apicola and interwoven with Lactobacillus Firm5 [10,19 $\left.{ }^{\bullet \bullet}, 26\right]$. Slightly upstream, near the point source for host waste excretion, a narrow ring of pyloric epithelium is vulnerable to scab formation, a melanization immune response to Frischella perrara $\left[27^{\bullet \bullet}\right]$. Adapted for biofilm life, the ileum species are equipped to cooperate with, but also exploit eukaryotic and bacterial cells. They are enriched for carbohydrate metabolism, possess various secretion systems, known bacteriocins, and other functional systems to kill close competitors under conditions of nutrient limitation (iron scavenging), DNA damage, or oxidative stress $[8,28$ 30]. The core hindgut species Lactobacillus Firm4, L. Firm5 and Bifidobacterium play functionally different roles, consistently dominate the rectum, and outnumber the ileum bacteria by an order of magnitude [26]. Amplicon samples of whole guts reflect this numeric difference often obscuring bacterial community structure in the information-rich ileum. Targeted by a couple amplicon studies, the midgut contains a magnitude less bacteria than the ileum, and the crop contains the least (Figure 1). 
Bacterial communities (16S amplicons) from distinct studies and sample types including whole gut samples of workers, queens and drones (males), five worker tissue types, worker guts by age, and niches associated with foraging, nursing and food storage.

\section{ADULT WORKER HINDGUT (CORE) \\ Lactobacillus firm5 \\ Lactobacillus firm4 \\ Snodgrassella alvi \\ Gilliamella apicola \\ Bifidobacterium spp. \\ Frischella perarra}

\section{LESSER KNOWN GUT-ASSOCIATED}

Bartonella apis Acetobacteraceae Alpha 2.1

\section{HIVE AND SOCIAL ENVIRONMENT}

Lactobacillus kunkeei Xanthomonadaceae Parasaccharibacter apium Actinomycetales

\section{OTHER BACTERIA}

Melissococcus plutonius (EFB, larval pathogen) Environmental source or unrecognized symbiont

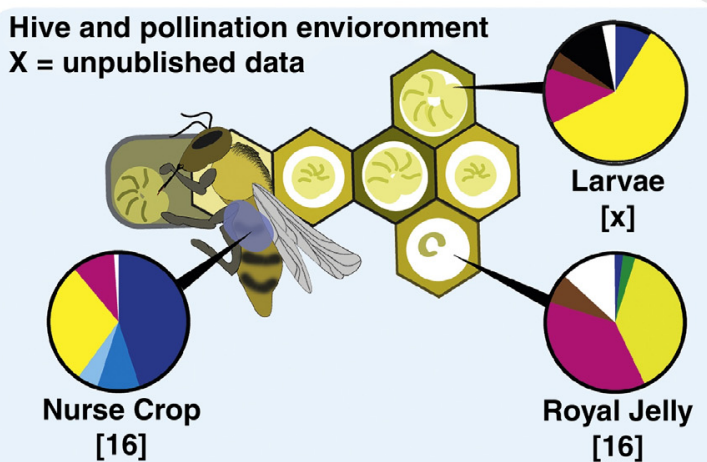

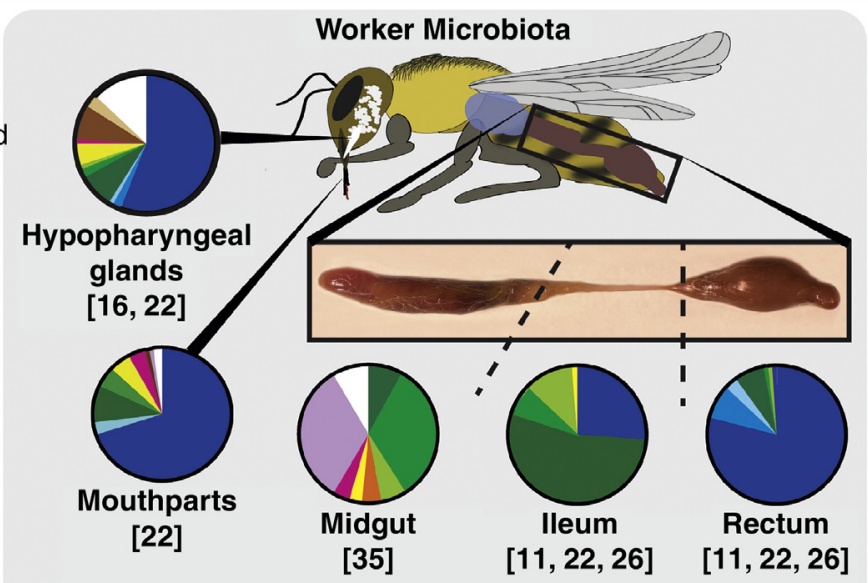

Bacterial communities from worker bee tissues associated with royal jelly secretion [16,22], food sharing [22], digestion and nutrient absorption [35], host waste excretion (ileum) and ion reuptake (rectum) $[11,22,26]$.

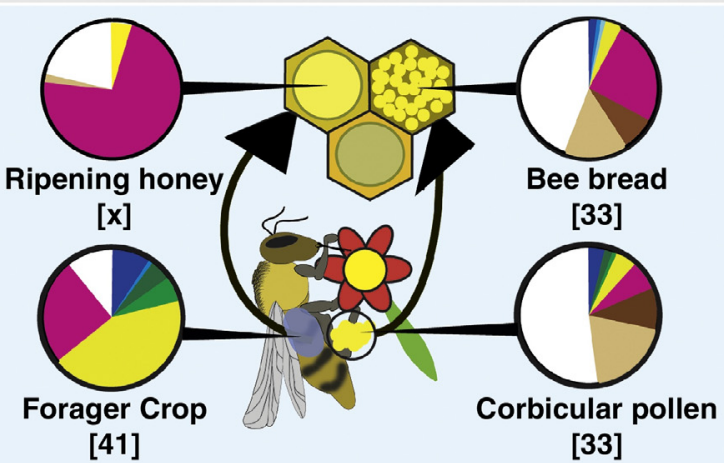

From the microbial perspective, the hive of the honey bee represents the devil and the deep blue sea, teeming with nutrients, antimicrobial substances and hygienic behaviors [54]. The name sake honey, comes with a persistent source of $\mathrm{H}_{2} \mathrm{O}_{2}$, low $\mathrm{pH}$ (3-4), crippling desiccation potential and immune factors [55]. Collected pollen comes with its own protective shell, but for good measure, is mixed with $40-50 \%$ honey [41], which aids in preservation [33]. Pollen is consumed by nurse workers and converted to royal jelly, a complete nutrition spiked with antibacterial and immunity proteins [56]. Secreted by glands in the nurse worker, royal jelly [16] is the diet of queens and newly emerged larvae [16], but also provided to begging foragers and perhaps drones. Antimicrobial plant resins are collected and distributed throughout the hive (propolis) decreasing the cost of individual immunity [57]. In this extreme environment, the core gut bacteria are transmitted across generations, specialized pathogens await opportunity, and hive bacteria make a living.

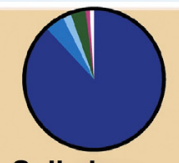

Cell cleaner

[38]

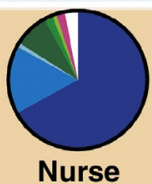

[45]

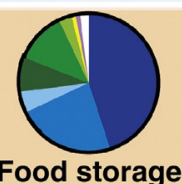

[13]

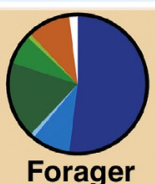

$[41,45,46]$

lleum specific and olher gram negative bacteria accrue with age. Gut bactenal communities retrieved from whole worker guts sampled according to known age, behavior in the hive or age-related task [40]. Task: age range; Cell cleaner: 0-2 days [381, Nurse: $2-11$ days [45] Food storage: $11-20$ days [13], Forager: $20+$ days $[41,45,46)$.
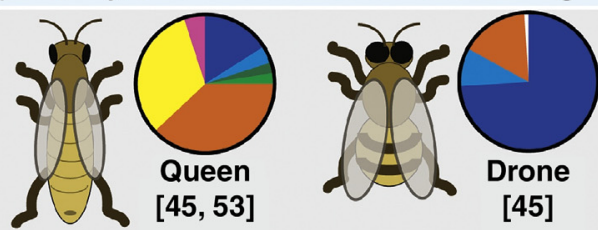

Gut bacteria in the reproductive caste differs from workers. Drones [45] are ephemeral, but queens, apparently dominated by Acetobacteraceae [45,53], can live for many years and are fed royal jelly throughout their lives to support egg laying.

Current Opinion in Insect Scienc

Bacterial communities [16S amplicons] from distinct studies and sample types including whole gut samples of workers, queens and drones (males), five worker tissue types, worker guts by age, and niches associated with foraging, nursing and food storage [57]. 
Core gut bacterium $S$. alvi has high fidelity for the host ileum, and many of its secretion systems have co-evolved to interface peacefully with host epithelium $\left[19^{\bullet \bullet}\right]$. S. alvi derives energy from the oxidation of carboxylates so can benefit from the fermentation products of neighboring bacteria $\left[9^{\bullet \bullet}\right]$. S. alvi secretes and produces nutrients needed by other gut and hive bacteria, so represents exploitable resource potential for oxygen tolerant opportunists [10]. Experiments with mutant $S$. alvi strains have shown that biofilm function relies in part on secreted siderophores to scavenge iron [ $\left[9^{\bullet \bullet}\right]$. Iron is often depleted in gut bacteria but is necessary for defusing superoxide ions generated by aerobic respiration. Other bacterial genomes from the hive and gut are equipped for iron scavenging but do not secrete siderophores, suggesting a variety of microbes may exploit $S$. alvi if its biofilm partners (G. apicola and L. firm5) do not properly establish. Genomic and metagenomic analysis of $G$. apicola and $S$. alvi have cast their relationship as complimentary, harboring functions enriched for intracellular communication [28,29]. Curiously, we find little abundance relationship between $S$. alvi and G. apicola in amplicon studies of early adults. This relationship changes with adult age (Figure 1). As determined for Apis cerana [31], G. apicola may be better established at middle age, prior to foraging. Sequencing of individual G. apicola cells reveals high diversity in accessory gene sets by strain suggesting adaptation to a wide variety of honey bee niches [32]. $G$. apicola is found throughout the hive environment and is closely related to strains from the pollination environment $[18,33]$. The midgut is often dominated by $G$. apicola $[34,35]$. Collectively, these results suggest broad ecology of G. apicola strains in need of functional and taxonomic refinement. The close cooperation hypothesized for $S$. alvi and G. apicola combined with deep functional variation in G. apicola suggests the evolution of social cheaters that exploit the biofilm environment and diminish its structure $\left[19^{\bullet \bullet}, 36\right]$.

\section{Gut bacterial succession in adult workers}

The formation, persistence and protective qualities of the gut microbiota are critical for normal host function $\left[22^{\bullet \bullet}, 37\right]$. Generational transmission of core gut bacteria is controversial in honey bees, hypothesized to rely on social contact with older adults $\left[9^{\bullet}, 11\right]$. However all core gut bacterial groups are acquired from hive materials within a few hours of exposure $\left[22^{\bullet \bullet}, 38\right]$. In one study, adult gut bacterial structure at 3 days, and bacterial succession from 3 to 8 days were unaffected by early contact with older adults [38]. All adult gut bacteria can be found in larvae $[15,31,39]$ that then defecate in their sealed cell prior to pupation. Adults (both workers and queens) likely emerge with core gut bacteria in or on their bodies. Post emergence, the first worker role is cell cleaner, a task largely confined to the brood area [40]. Alternatively, 'gut defecate bombs' are often detected in corbicular pollen or stored pollen, and core gut bacteria can be revived from a variety of hive materials [33,41]. Common garden experiments indicate host fidelity of native core gut strains can overcome a large numerical disadvantage when populating their native host [6]. Although scarce in the hive environment relative to other gut bacteria, $S$. alvi quickly populates newly emerged adults, a testament to host fidelity $[11,38]$. S. alvi, L. firm5, L. firm 4 and Bifidobacterium are all high-fidelity gut colonizers populating the hindgut by 3 days of age $\left[20^{\bullet}, 22^{\bullet \bullet}, 24^{\bullet}\right]$. Most speciose and abundant throughout the alimentary tract, L. Firm 5 accounts for most of the total gut bacteria in 3 -day old worker guts $\left[22^{\bullet \bullet}, 38\right]$.

Bacterial abundance in whole guts tops out as bees begin to consume pollen 3 days post-adult emergence, but sweeping taxonomic shifts occur from 3 to 8 days suggesting pioneer or niche construction strains $[11,38,42]$. While the rectum community seems to possess an emergent structure by 3 days of age, the ileum of the early adult bee seems a more turbulent niche with structure emerging after 8 days [11]. Much of this may depend on the prevalence of core ileum species G. apicola and F. perrara, associated host immune response and successional alteration of the ileum environment $\left[27^{\bullet \bullet}, 43^{\bullet \bullet}\right]$. Colonizing ileum epithelial tissue, $S$. alvi is largely tolerated by the host eliciting a minor immune response $\left[20^{\bullet}, 43^{\bullet \bullet}\right]$. By contrast, the host resists $F$. perrara colonization mounting a major immune response and melanization cascade, suggesting that $F$. perrara is a newly evolved and/or recently acquired symbiont $\left[17^{\bullet \bullet}, 43^{\bullet \bullet}\right]$. Both host immune expression (antimicrobial peptides AMPs) and gut niche alteration (ROS) are implicated in shaping early adult microbiota $\left[20^{\circ}, 43^{\bullet \bullet}\right]$. Host protection may also rely on the degree of partner fidelity between established $S$. alvi and introduced G. apicola $\left[1^{\circ} \cdot\right.$. This in turn might depend on social exposure to multiple G. apicola strains, increasing the chance for an encounter with a compatible strain. Providing newly emerged bees with a cocktail of pre-established gut communities rapidly results in greater community evenness, including the early representation of G. apicola in young adults [11]. This suggests that the introduction of a community of compatible strains already shaped and structured with respect to one another and host physiology may facilitate or greatly accelerate the process of community establishment.

Similar to gut bacterial succession in Apis cerana [31] the compositional shift from young to old adult in Apis mellifera involves a continual reduction of Lactobacillus (Figure 1). Early adult enterotypes are dominated by core Lactobacillus that yields to Gammaproteobacteria and Acetobacteraceae over the life of the bee (Figure 1). The Drosophila gut ages similarly. Unhealthy function in Drosophila is tightly linked to an age-related decrease in Lactobacillus and subsequent increases in Gammaproteobacteria and Acetobacteraceae [44]. In the oldest honey bees (foragers), Acetobacteraceae Alpha 2.1 begins to 
appear uniformly across individuals $[41,45,46]$. In one forager study, whole guts contained $>65 \%$ Gammaproteobacteria uniformly across individuals from many colonies [46]. In two separate studies and seasons, forager honey bee guts were uniformly Lactobacillus dominant suggesting different methods or environmental factors $[41,45]$. It is unknown if extreme depletion of Lactobacillus in foragers represents dysbiosis or a normal senescence enterotype (see [46]).

\section{Worker gut dysbiosis}

It is clear that an understanding of healthy gut function in Apis mellifera requires consideration of host life stage and tissue-specific bacterial succession $\left[11,22^{\bullet \bullet}, 31,38,43^{\bullet \bullet}\right]$. Only a few experiments have paired host function to a shifting microbiota or particular bacterial strain (Table 1). The establishment of a typical gut microbiota leads to greater weight gain [10], while early adult dysbiosis is associated with low adult weight, deficient worker development and early mortality [22 $\left.2^{\bullet}\right]$. Assuming that shifts in the relative abundance of particular groups and species are strongly associated with health or disease, we take liberties discussing adult gut dysbiosis in honey bees as a compositional or membership departure from whole gut samples of healthy workers (Figure 1). The characterization of gut bacterial function is progressing rapidly in honey bee workers $\left[10,19^{\bullet \bullet}, 20^{\bullet}, 22^{\bullet \bullet}, 24^{\bullet}, 43^{\bullet \bullet}\right]$. Whether incidental or part of a controlled study, induced gut dysbiosis provides a rich context to understand the contribution of community structure to disease progression and early host senescence. Below we discuss two potentially emergent types of dysbiosis; altered structure characterized by shifts among core gut species, and complete displacement of the core gut bacteria by opportunistic hive bacteria and known disease agents.

\section{Gut dysbiosis (altered structure)}

A recent induction of gut dysbiosis revealed that community structure can be irreversibly perturbed in early adults, and underscores the importance in functional studies of obtaining tissue-specific $16 \mathrm{~S}$ amplicon data to reveal gut community structure $\left[7^{\bullet}, 37\right]$. Similar across four recent studies, the increased prevalence of core gut species G. apicola in early adults was associated with dysbiosis and host deficiencies $\left[22^{\bullet \bullet}, 24^{\bullet}, 37,47\right]$. In our putatively dysbiotic foragers [46], increases of core $G$. apicola and other non-core species seemed to have displaced many core gut bacteria suggesting diminished or alternate biofilm function. Similarly, in-hive exposure to a broad spectrum antibiotic increased the prevalence of $G$. apicola in worker guts [47]. Bees treated with tetracycline during early adult succession show reduced survivorship associated with gut dysbiosis, characterized by increased abundance and diversity of $G$. apicola $\left[24^{\circ}\right]$. Thus, if increases in $G$. apicola commonly occur with dysbiosis or age-related succession, this pattern may be difficult to distinguish from beekeeper treatment or agricultural exposure.

More detailed results on early adult dysbiosis come from deep sequencing of specific tissues associated with social contact and digestion $\left[22^{\bullet \bullet}\right]$. Here, dysbiosis was detected simultaneously in the mouth, hypopharyngeal glands, ileum, and rectum of stressed individuals. The communities associated with host deficiencies were characterized by shifts in the relative abundance of core ileum species as opposed to a loss of core rectum species. Deep sequencing of the ileum revealed extreme displacement of $S$. alvi by gut residents $F$. perrara and G. apicola with some help from hive opportunists P. apium and L. kunkeei. This shift was strongly associated with poor host development and early mortality, and correlated with reduced biofilm function and host tissue disruption by $F$. perrara and its associates $\left[22^{\bullet \bullet}, 27^{\bullet \bullet}\right]$. Host melanization in response to $F$. perrara is accompanied by major oxidative stress in the gut environment $\left[27^{\circ \bullet}\right]$, perhaps facilitating the invasion of oxygen tolerant opportunists from other hive or gut niches.

\section{Gut dysbiosis (complete displacement)}

A wealth of information on dysbiosis comes from a one month confinement of colonies to small flight enclosures [21]. Indoor colonies quickly decline and often develop disease. Confinement to greenhouses causes increased Nosema disease in the midgut, premature worker senescence and immune-suppression associated with oxidative stress [48]. Confinement trials from separate years each reveal unique dysbiotic gut profiles. Some of these snapshots in time seem to have captured the progression of dysbiosis, but most worker gut bacterial communities were completely dominated by a single species. The first trial was marked by blooms of Bartonella apis in many individuals. Well equipped for host-gut interaction, $B$. apis dominated the guts of two workers displacing all core ileum species. Members of this genus are well known opportunists, seemingly harmless commensals of ants, but little is known for bees. Considered a 'core gut bacteria', broad phylogenetic analyses suggest $B$. apis is specific to genus Apis $\left[17^{\bullet \bullet}, 49\right]$, but it is rarely seen in worker gut samples. It was however, detected in two separate midgut studies, one at uniformly high relative abundance [35], and the other as a seasonal or diet-induced bloom [34]. $B$. apis seems to exploit the stressed, young, and old bees showing sporadic abundance in whole guts of newly emerged workers $\left[24^{\circ}\right]$, and occurring uniformly across our putatively dysbiotic foragers (see [46]).

A separate confinement experiment produced a completely different form of dysbiosis, characterized by a total lack of native gut bacteria in adults [21]. The core bacteria were displaced by specialized larval pathogen Melissococcus plutonius, the causative agent of European foulbrood, and associated secondary opportunists 
Enterococcus faecalis and Paenibacillus alvei [50]. Here again, most gut libraries were dominated by hive bacteria L. kunkeei or P. apium. In confined bees, each of these five species dominated at least one gut library. While it's tempting to consider this a final result, disease states are also typified by microbial succession. Thus, each amplicon snapshot might be interpreted as a different stage in disease progression. Each successive bacterial bloom may alter gut physiochemistry for the next in a sequence of species or strains. This is the first evidence that specialized larval pathogen $M$. plutonius and associated opportunists occur as dominance environments in the adult gut, completely displacing the native biofilm. In Apis cerana, larvae infected with $M$. plutonius show a diminished gut community typically associated with adult Apis [31]. Collectively these results suggest that adult guts may provide a breeding ground for larval disease under stressful conditions. Given the vast array of fringe environments associated with host developmental stages, food storage, and social behavior, the honey bee may host many opportunists and pathogens that employ a similar virulence strategy, cycling between refugial, transitional and target niches.

\section{Summary}

The stability of the worker microbiota suggests a healthy ecosystem, but like all well-structured communities, it is beset with cryptic cheaters and opportunists. Based on the above synthesis, we speculate that disease causing organisms compete and cooperate with hive associates $P$. apium and L. kunkeei over evolutionary time across multiple hive and social niches including the larval and adult gut. Under certain conditions, the worker host may tolerate the hive bacteria; they are commonly consumed at low levels and are more abundant throughout the hive system than are specialized pathogens. But following perturbation of core ileum species, these hive residents may act as secondary invaders, displacing the native gut microbiota. Oxygen dynamics in the gut [10] may facilitate this shift favoring invasion by more oxygen-tolerant microbes. A suite of ROS dynamics known from mammals [51] are predicted for honey bees including host contributions to biofilm structure $\left[20^{\circ}\right]$, and cell damage that accompanies dysbiosis. Complete displacement of the gut community may contribute to, or even represent the point source for many opportunistic disease states associated with honey bee colony decline.

Honey bee gut and hive bacteria are important to healthy host function, and their natural succession and dysbiotic states are revealing the nature of host protection and its association with disease ecology $\left[10,12,19^{\bullet \bullet}, 20^{\bullet}, 22^{\bullet \bullet}, 24^{\bullet}\right.$, $\left.37,43^{\circ}\right]$. The honey bee pathosphere is not clearly defined but depends on a collection of developmental and stochastic factors including the state of the host and the structure of its microbiota $\left[1^{\circ}\right]$. Within a well-structured gut microbiota, seemingly dominated by L. Firm $5 / L$.
Firm4/Bifidobacterium (rectum) and S. alvilG. apicola (ileum), the gut bacteria are benevolent protectors. When this structure is compromised, the protective symbiosis is diminished. Although early immune priming by $F$. perrara may prove beneficial, its' excessive growth damages the gut environment $\left[43^{\circ \bullet}\right]$, and it is strongly correlated

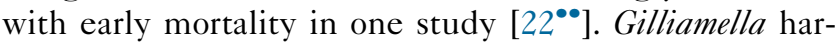
bors great diversity and may play many function roles including protector, opportunist and cheater $[6,31,51]$. Simulating and perturbing natural colonization of gut bacteria using combinations of gut and hive strains will provide further insight into community function [11,20 $\left.{ }^{\circ}\right]$. Finally, workers and queens seem to maintain different gut bacteria, but the lack of an emergent community structure in queens indicates more and deeper sampling is required to determine the core 'royal' microbiota $[45,53]$. Roll out the red carpet.

\section{Conflict of interest}

The authors declare no conflict of interest.

\section{Acknowledgements}

We thank Anderson lab members for related discussion and Dr. Adam Dolezal, Dr. Mark Carroll and two anonymous reviewers for comments that improved the manuscript. The corresponding author thanks Belynda Starr, Ariel Calypso and Isaak Edward for their valuable input. This work was supported by the USDA-ARS, an equal opportunity employer and provider.

\section{References and recommended reading}

Papers of particular interest, published within the period of review, have been highlighted as:

- of special interest

- of outstanding interest

1. Schwarz RS, Huang Q, Evans JD: Hologenome theory and the - $\quad$ honey bee pathosphere. Curr Opin Insect Sci 2015, 10:1-7. Summarizes the honey bee microbiota and pathosphere, calling for a systems approach. Highlights the importance of context in host-microbial interactions.

2. Hölldobler B, Wilson EO: The Superorganism: The Beauty, Elegance, and Strangeness of Insect Societies. W.W. Norton \& Company; 2008.

3. Moran NA, Sloan DB: The hologenome concept: helpful or $\bullet \quad$ hollow? PLoS Biol 2015, 13:1-10.

Dismisses the holobiont as overly inclusive and low utility, outlining patterns misinterpreted as co-evolution. Applies the context and predicted requirements of group selection to gut microbial evolution.

4. Levy M, Kolodziejczyk AA, Thaiss CA, Elinav E: Dysbiosis and the immune system. Nat Rev Immunol 2017, 17:219-232.

5. Lively CM, de Roode JC, Duffy MA, Graham AL, Koskella B: Interesting open questions in disease ecology and evolution. Am Nat 2014, 184:S1-S8.

6. Kwong WK, Moran NA: Evolution of host specialization in gut microbes: the bee gut as a model. Gut Microbes 2015, 6:214220.

7. Engel $P$, Kwong WK, Mcfrederick Q, Anderson KE, Barribeau M,

- $\quad$ Chandler JA, Cornman RS, Link C, Engel P, Kwong WK et al.: The bee microbiome: impact on bee health and model for evolution and ecology of host-microbe interactions. mBio 2016, 7:1-9. Promotes honey bees as a model for host-microbial interaction and ecology and evolution of gut microbiota. Outlines approaches for scientific advancement discussing challenges and resource management.

8. Moran NA: Genomics of the honey bee microbiome. Curr Opin Insect Sci 2015, 10:22-28. 
9. Kwong WK, Moran NA: Gut microbial communities of socia -• bees. Nat Rev Microbiol 2016, 14:374-384.

Excellent comprehensive review, with reference to predicted genome function and metatranscriptomics of core gut bacterial groups. Outlines a hypothesis for gut biofilm interaction and niche partitioning.

10. Zheng H, Powell JE, Steele MI, Dietrich C, Moran NA: Honeybee gut microbiota promotes host weight gain via bacterial metabolism and hormonal signaling. Proc Natl Acad Sci 2017 http://dx.doi.org/10.1073/PNAS.1701819114.

11. Powell JE, Martinson VG, Urban-Mead K, Moran NA: Routes of acquisition of the gut microbiota of the honey bee Apis mellifera. Appl Environ Microbiol 2014, 80:7378-7387.

12. Schmidt $K$, Engel $P$ : Probiotic treatment with a gut symbiont leads to parasite susceptibility in honey bees. Trends Parasitol 2016, 32:914-916.

13. Moran NA, Hansen AK, Powell JE, Sabree ZL: Distinctive gut microbiota of honey bees assessed using deep sampling from individual worker bees. PLOS One 2012, 7:1-10.

14. Otto M: Staphylococcus epidermidis - the 'accidental' pathogen. Nat Rev Microbiol 2009, 7:555-567.

15. Vojvodic S, Rehan SM, Anderson KE: Microbial gut diversity of Africanized and European honey bee larval instars. PLOS One 2013, 8:e72106.

16. Corby-Harris V, Snyder LA, Schwan MR, Maes P, McFrederick QS Anderson KE: Origin and effect of Alpha 2.2 Acetobacteraceae in honey bee larvae and description of Parasaccharibacter apium gen. nov., sp. nov. Appl Environ Microbiol 2014, 80:7460-7472.

17. Kwong WK, Medina LA, Koch H, Sing K-W, Soh EJY, Ascher JS,

-• Jaffé R, Moran NA: Dynamic microbiome evolution in social bees. Sci Adv 2017, 3:1-17.

Charles Duncan Michener would be proud. Evolution of gut microbiota from eusocial bees sampled around the globe. Patterns of diversification were associated with colony size suggesting that the gut microbiota was shaped in part by selection above the level of the individual.

18. McFrederick QS, Thomas JM, Neff JL, Vuong HQ, Russell KA, Hale AR, Mueller UG: Flowers and wild megachilid bees share microbes. Microb Ecol 2016 http://dx.doi.org/10.1007/s00248016-0838-1.

19. Powell JE, Leonard SP, Kwong WK, Engel P, Moran NA: Genome-

-. wide screen identifies host colonization determinants in a bacterial gut symbiont. Proc Natl Acad Sci U S A 2016, 113:13887-13892.

Systems approach exemplar. Experiments introducing bacterial mutants revealed a wealth of genomic information about biofilm formation. Briefly discussed biofilm cheater evolution.

20. Kwong WK, Mancenido AL, Moran NA: Immune system

- $\quad$ stimulation by the native gut microbiota of honey bees. $R$ Soc Open Sci 2017, 4

Demonstrates differential expression of host genes in response to core gut bacteria. Discussed the influence of host immune response and reactive oxygen species on biofilm integrity.

21. DeGrandi-Hoffman G, Corby-Harris V, DeJong EW, Chambers M, Hidalgo G: Honey bee gut microbial communities are robust to the fungicide Pristine ${ }^{\circledR}$ consumed in pollen. Apidologie 2016 http://dx.doi.org/10.1007/s13592-016-0478-y.

22. Maes PW, Rodrigues PAP, Oliver R, Mott BM, Anderson KE: Diet-

-• related gut bacterial dysbiosis correlates with impaired development, increased mortality and Nosema disease in the honeybee (Apis mellifera). Mol Ecol 2016, 25:5439-5450.

Statistically robust deep-sequencing experiment reveals that host deficiencies are strongly correlated with early adult dysbiosis. Multi-tissue sampling shows dysbiosis manifests systemically throughout the host.

23. Cariveau DP, Powell JE, Koch H, Winfree R, Moran NA: Variation in gut microbial communities and its association with pathogen infection in wild bumble bees (Bombus). ISME J 2014, 8:2369-2379.

24. Raymann K, Shaffer Z, Moran NA: Antibiotic exposure perturbs the - gut microbiota and elevates mortality in honeybees. $2016 \mathrm{http} / / /$ dx.doi.org/10.1371/journal.pbio.2001861.

Gut dysbiosis, marked by increased G. apicola, results from host exposure to an antibiotic commonly used by beekeepers. Antibiotic exposure increased susceptibility to opportunistic pathogens resulting in significantly greater bee mortality.

25. Hillman K, Goodrich-Blair H: Are you my symbiont? Microbial polymorphic toxins and antimicrobial compounds as honest signals of beneficial symbiotic defensive traits. Curr Opin Microbiol 2016, 31:184-190.

26. Martinson VG, Moy J, Moran NA: Establishment of characteristic gut bacteria during development of the honeybee worker. Appl Environ Microbiol 2012, 78:2830-2840.

27. Engel $P$, Bartlett KD, Moran NA: The bacterium Frischella -. perrara causes scab formation in the gut of its honey bee host. MBio 2015 http://dx.doi.org/10.1128/mBio.00193-15.

Strong causal demonstration that the long-known scab phenotype in the bee gut is due to ileum/pylorus resident Frischella perrara. Resultant immune response and melanization alters the host gut environment, but may also prime the immune system.

28. Kwong WK, Engel P, Koch H, Moran NA: Genomics and host specialization of honey bee and bumble bee gut symbionts. Proc Natl Acad Sci 2014, 111:11509-11514.

29. Engel P, Martinson VG, Moran NA: Functional diversity within the simple gut microbiota of the honey bee. Proc Natl Acad Sci U S A 2012, 109:1-6.

30. Lee FJ, Rusch DB, Stewart FJ, Mattila HR, Newton ILG: Saccharide breakdown and fermentation by the honey bee gut microbiome. Environ Microbiol 2015, 17:796-815.

31. Guo J, Wu J, Chen Y, Evans JD, Dai R, Luo W, Li J: Characterization of gut bacteria at different developmenta stages of Asian honey bees, Apis cerana. J Invertebr Pathol 2015, 127:110-114.

32. Engel P, Stepanauskas R, Moran NA: Hidden diversity in honey bee gut symbionts detected by single-cell genomics. PLOS Genet 2014. doi.org/10.1371/journal.pgen.1004596.

33. Anderson KE, Carroll MJ, Sheehan TIM, Mott BM: Hive-stored pollen of honey bees: many lines of evidence are consistent with pollen preservation, not nutrient conversion. $\mathrm{Mol}$ Ecol 2014 http://dx.doi.org/10.1111/mec.12966.

34. Ludvigsen J, Rangberg A, Avershina E, Sekelja M, Kreibich C, Amdam G, Rudi K: Shifts in the midgut/pyloric microbiota composition within a honey bee apiary throughout a season. Microbes Environ 2015, 30:235-244.

35. Jia H-R, Li-Li G, Li Y-H, Wang Q, Diao Q-Y, Zhou T, Dai P-L: The effects of Bt Cry1le toxin on bacterial diversity in the midgut of Apis mellifera ligustica (Hymenoptera: Apidae). Sci Rep 2016 http://dx.doi.org/10.1038/srep24664.

36. Hibbing ME, Fuqua C, Parsek MR, Peterson SB: Bacterial competition: surviving and thriving in the microbial jungle. Nat Rev Microbiol 2010, 8:15-25.

37. Schwarz RS, Moran NA, Evans JD: Early gut colonizers shape parasite susceptibility and microbiota composition in honey bee workers. Proc Natl Acad Sci U S A 2016, 113:9345-9350.

38. Anderson KE, Rodrigues PAP, Mott BM, Maes P, Corby-Harris V: Ecological succession in the honey bee gut: shift in Lactobacillus strain dominance during early adult development. Microb Ecol 2016, 71:1008-1019.

39. Hroncova Z, Havlik J, Killer J, Doskocil I, Tyl J, Kamler M, Titera D, Hakl J, Mrazek J, Bunesova V et al.: Variation in honey bee gut microbial diversity affected by ontogenetic stage, age and geographic location. PLOS One 2015, 10:1-17.

40. Seeley $D$, Kolmes A: Age polyethism for hive duties in honey bees - illusion or reality? When marked with paint for behavioral studies, newly emerged honeybees return invariably to the brood area. Ethology 1991, 87:284-297.

41. Corby-Harris V, Maes P, Anderson KE: The bacterial communities associated with honey bee (Apis mellifera) foragers. PLOS One 2014, 9.

42. Rokop ZP, Horton MA, Newton ILG: Interactions between cooccurring lactic acid bacteria in honey bee hives. Appl Environ Microbiol 2015, 81:7261-7270. 
43. Emery O, Schmidt K, Engel P: Immune system stimulation by the -• gut symbiont Frischella perrara in the honey bee (Apis mellifera). Mol Ecol 2017 http://dx.doi.org/10.1111//mec.14058. Detailed analysis of host expression in response to controlled introductions of ileum natives F. perrara and S. alvi. The honey bee largely tolerates S. alvi, but mounts a strong immune response to $F$. perrara. Results highlight the role of the host immune system in regulating gut bacterial symbiosis.

44. Clark RI, Salazar A, Yamada R, Fitz-Gibbon S, Morselli M, Alcaraz J, Rana A, Rera M, Pellegrini M, Ja WW et al.: Distinct shifts in microbiota composition during Drosophila aging impair intestinal function and drive mortality. Cell Rep 2015, 12:1656-1667.

45. Kapheim KM, Rao VD, Yeoman CJ, Wilson BA, White BA, Goldenfeld N, Robinson GE: Caste-specific differences in hindgut microbial communities of honey bees (Apis mellifera). PLoS One 2015, 10:1-14.

46. Horton MA, Oliver R, Newton IL: No apparent correlation between honey bee forager gut microbiota and honey production. PeerJ 2015, 3:e1329.

47. Kakumanu ML, Reeves AM, Anderson TD, Rodrigues RR, Williams MA: Honey bee gut microbiome is altered by in-hive pesticide exposures. Front Microbiol 2016, 7:1-11.

48. Morimoto T, Kojima Y, Toki T, Komeda Y, Yoshiyama M, Kimura K, Nirasawa K, Kadowaki T: The habitat disruption induces immune-suppression and oxidative stress in honey bees. Ecol Evol 2011, 1:201-217.

49. Kešnerová L, Moritz R, Engel P: Bartonella apis sp. nov., a honey bee gut symbiont of the class Alphaproteobacteria. Int J Syst Evol Microbiol 2016, 66:414-421.
50. Forsgren E: European foulbrood in honey bees. J Invertebr Pathol 2010, 103:S5-S9.

51. Alenberg L, Espinova T, Judge C, Bittinger K, Chen J, Al E: Correlation between intraluminal oxygen gradient and radial partitioning of intestinal microbiota in humans and mice. Gastroenterology 2015, 147:1055-1063.

52. Zheng H, Nishida A, Kwong WK, Koch H, Engel P, Steele MI, Moran NA: Metabolism of toxic sugars by strains of the bee gut symbiont Gilliamella apicola. MBio 2016, 7:e01326-e1416.

53. Tarpy DR, Mattila HR, Newton ILG: Development of the honey bee gut microbiome throughout the queen-rearing process. Appl Environ Microbiol 2015, 81:3182-3191.

54. Evans JD, Spivak M: Socialized medicine: individual and communal disease barriers in honey bees. $J$ Invertebr Pathol 2010, 103:S62-S70

55. Vannette RL, Mohamed A, Johnson BR: Forager bees (Apis mellifera) highly express immune and detoxification genes in tissues associated with nectar processing. Sci Rep 2015, 5:16224.

56. Fontana R, Mendes MA, De Souza BM, Konno K, César LMM, Malaspina O, Palma MS: Jelleines: a family of antimicrobial peptides from the Royal Jelly of honeybees (Apis mellifera). Peptides 2004, 25:919-928.

57. Simone M, Evans JD, Spivak M: Resin collection and social immunity in honey bees. Evolution 2009, 63:3016-3022. 